Interfacial deflection and jetting of a paramagnetic particle-laden fluid: theory and experiment

by

Scott S. H. Tsai

lan M. Griffiths

Zhenzhen Li

Pilnam Kim

Howard A. Stone 



\title{
Interfacial deflection and jetting of a paramagnetic particle-laden fluid: theory and experiment
}

\author{
Scott S. H. Tsai, ${ }^{\star a b}$ Ian M. Griffiths, ${ }^{\star c}$ Zhenzhen Li, $^{a \dagger}$ Pilnam Kim, ${ }^{a \ddagger}$ and Howard A. Stone ${ }^{\star a}$ \\ Received Xth $X X X X X X X X X X 20 X X$, Accepted Xth $X X X X X X X X X 20 X X$ \\ First published on the web Xth $X X X X X X X X X X 200 X$ \\ DOI: 10.1039/b000000x
}

We describe the results of experiments and mathematical analysis of the deformation of a free surface by an aggregate of magnetic particles. The system we study is differentiated from ferrofluid systems because it contains regions rich with magnetic material as well as regions of negligible magnetic content. In our experiments, the magnetic force from a spherical permanent magnet collects magnetic particles to a liquid-air interface, and deforms the free surface to form a hump. The hump is composed of magnetic and non-magnetic regions due to the particle collection. When the magnet distance falls below a threshold value, we observe the transition of the hump to a jet. The mathematical model we develop, which consists of a numerical solution and an asymptotic approximation, captures the shape of the liquid-air interface during the deformation stage and a scaling prediction for the critical magnet distance for the hump to become a jet.

\section{Introduction}

Magnetic nano- and microparticles arise in a wide range of applications. Functionalized magnetic microparticles, in the form of bulk dilute suspensions, are used to perform separations of biological material such as cells ${ }^{1,2}$ and can act as forcing elements for microrheology. ${ }^{3-5}$ Recently, similar magnetic microparticles have been used in microfluidic devices for cell sorting, ${ }^{6-8}$ blood cleansing, ${ }^{9}$ interfacial tensiometry, ${ }^{10}$ and magneto-capillary self-assembly. ${ }^{11}$

When magnetic nanoparticles such as magnetite are suspended at high concentration in aqueous or non-aqueous carrier-fluids, the entire system behaves as a continuum of magnetic fluid. ${ }^{12}$ The rheology and interfacial shape of these so-called ferrofluids can be tuned with external magnetic fields, and such systems have been studied extensively. ${ }^{13-18}$ The control of ferrofluid properties using magnetic fields have applications in mechanical sealing and acoustics, ${ }^{19}$ as well as in non-traditional areas such as the folding of elastic material $^{20}$ and controlled drug release. ${ }^{21}$

In the water-purification industry, where heavy-metal removal from contaminated water has been a formidable chal-

\footnotetext{
${ }^{a}$ Department of Mechanical and Aerospace Engineering, Princeton University, Princeton, New Jersey, USA. Email: hastone@princeton.edu

${ }^{b}$ Department of Mechanical and Industrial Engineering, Ryerson University, Toronto, Ontario, Canada.

${ }^{c}$ Mathematical Institute, University of Oxford, Oxford, England, United Kingdom.

† Present address: Laboratoire MMN, ESPCI ParisTech, Paris, France.

$\ddagger$ Present address: Department of Bio and Brain Engineering, KAIST, Daejeon, Republic of Korea.

$\star$ These authors contributed equally to this work.
}

lenge, ${ }^{22}$ a promising technique uses magnetic nanoparticles to remove heavy metals, such as arsenic, from water. Through adsorption, arsenic attaches to magnetic nanoparticles that are dispersed in the contaminated water, ${ }^{23}$ and the magnetically tagged clusters are collected with a magnetic field gradient. ${ }^{24}$ One possible mechanism for the safe collection and transfer of these magnetic aggregates out of the water is to use a magnetic field to pull the aggregates to the liquid-air interface, allow these aggregates to deform the interface, and eventually transition into a jet to remove the magnetic material.

In this paper, we describe experiments that are motivated by the industrial applications of forming and collecting aggregates of magnetic material. The system we study differs from a pure ferrofluid system because it contains both magnetically dominated and non-magnetic regions. Here, colloidal magnetic particles are first mixed with water, then collected at a liquid-air interface by an applied magnetic field from a permanent magnet (see Fig. 1).

We find that, when the permanent magnet is held suitably far from the fluid at a constant distance, the deflection of the liquid-air interface is generally static. However, as we reduce the distance, $L^{*}$, between the magnet and the unperturbed fluid interface, we find that there exists a threshold value below which the hump destabilizes and forms a jet (see Fig. 2).

The paper is organised as follows. In $\S 2$ we begin by describing the experimental methodology. In $\S 3$ we derive a mathematical model to describe the collection and arrangement of paramagnetic particles within the fluid beneath the external magnet, and the stable interfacial profile of the particle and fluid mixture that results. We compare the predictions made by this model with the experimental data. The complex- 


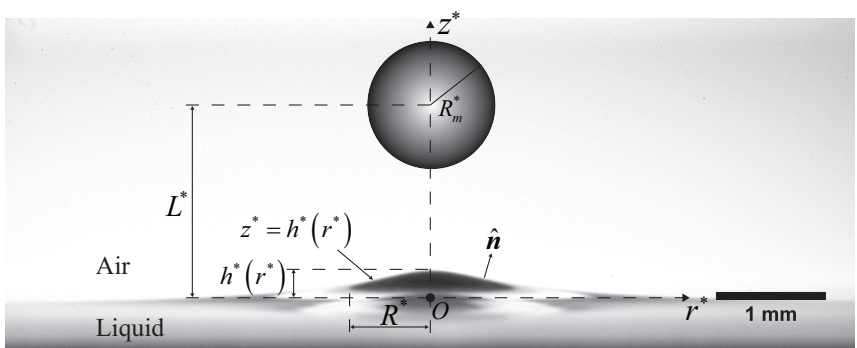

Fig. 1 An image from the magnetic hump formation experiment (with a drawing of the spherical magnet overlaid). The permanent magnet is placed a distance $L^{*}$ from the flat interface. Magnetic microparticles collect below the magnet and deform the liquid-air interface. The region where the magnetic particles aggregate is bounded by a base radius $R^{*}$.

ity of this theory is reduced in $\S 4$ by exploiting the typical small interfacial deflection of the fluid when the system is in a stable configuration. The simplified model lends itself to a tractable analysis of the system behaviour. In $\S 5$ we derive a scaling law utilizing the theoretical model that predicts when jetting of the fluid will occur. The simple trend from the scaling law allows the experimental data to be collapsed onto a single power-law curve. Finally, in $\S 6$ we draw conclusions on the model predictions and their application to real-world situations such as water purification.

\section{Experimental methods}

In our experiments, magnetic particles are collected at a liquid-air interface by the magnetic field gradient from a spherical permanent magnet. Fig. 1 shows an experimental image of a magnetic hump that is formed at the liquid-air interface, overlaid with a drawing of the spherical permanent magnet. (The actual magnet is too far from the hump to be included in Fig. 1.)

We plasma treat the back face of a $35 \mathrm{~mm}$ diameter petridish to make it hydrophilic. The back face of the petri-dish has $1 \mathrm{~mm}$ high walls that circle around the substrate to form a shallow reservoir. We deposit magnetic mixtures into this reservoir in all of our experiments.

In the interfacial deformation experiments, $1 \mathrm{~mL}$ of deionized (DI) water, with density $\rho=1,000 \mathrm{~kg} / \mathrm{m}^{3}$ and viscosity $\eta_{w}=1 \mathrm{mPa}$ s, is first deposited into the reservoir. A $1 \mu \mathrm{L}$ solution of water-based ferrofluid (EMG805, Ferrotec, Tokyo, Japan) is then injected into the reservoir using a micropipette to form the magnetic mixture. Here the ferrofluid is composed of $3.6 \%$ vol. of $10 \mathrm{~nm}$ paramagnetic nanoparticles, suspended in a DI water solution by anionic surfactants. Except for magnetic effects, we treat the magnetic mixtures as having the properties of water in all of our analysis because of the low

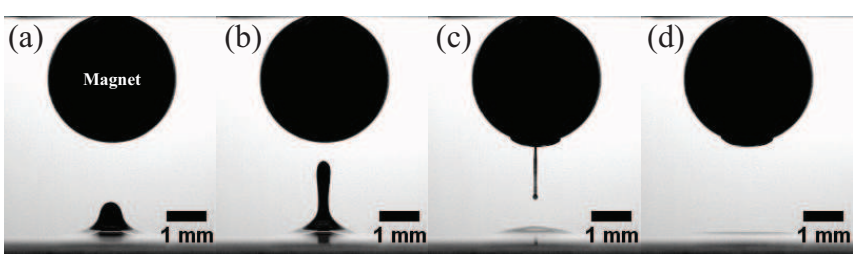

Fig. 2 A set of images, taken at $50 \mathrm{~ms}$ intervals, of the magnetic hump-jet transition. As the permanent magnet becomes sufficiently close to the magnetic hump, the hump (a) destabilizes, and (b)-(d) transitions to a jet.

concentration of ferrofluid used. (The ferrofluid has density $\rho_{m}=1,200 \mathrm{~kg} / \mathrm{m}^{3}$, and viscosity $\eta_{m}=3 \mathrm{mPa} \mathrm{s}$.)

In the jet formation experiments, magnetic particles (Sigma-Aldrich, St. Louis, MI, USA) of radius $5 \mu \mathrm{m}$, magnetic susceptibility $\chi \approx 10^{-3}$, and suspended in an aqueous buffer solution at a concentration $10^{12}$ beads $/ \mathrm{mL}$, are used in place of the ferrofluid for the magnetic mixture. We add the surfactant sodium dodecyl sulfate (SDS, Sigma-Aldrich, St. Louis, MI, USA) to the water in some experiments prior to mixing with magnetic particles to vary the interfacial tension, $\gamma$, and we measure the interfacial tension by the pendant drop method.

Spherical permanent magnets (K. J. Magnetics, Jamison, PA, USA), with radii $R_{m}^{*}=2.4$ or $3.2 \mathrm{~mm}$ and magnetization $M^{*} \approx 10^{6} \mathrm{~A} / \mathrm{m}$, are used to supply the magnetic field. In each experiment, a single magnet is super-glued to the tip of a plastic syringe, which is mounted to a motorized positioner. The positioner allows us to control the distance, $L^{*}$, between the magnet and the undeformed liquid interface (see Fig. 1).

The magnet is slowly brought to the vicinity of the magnetic fluid mixture at the beginning of each experiment, after which, the entire system is allowed sufficient time (approximately 10 minutes) for the particles to aggregate and equilibrate to a static hump at the liquid-air interface. We observe a distinct boundary that separates the region with magnetic material and the region outside, where there are no magnetic particles. In the interfacial deformation experiments, we measure a base radius $R^{*}$ (Fig. 1), of the magnetic region, and track the position of the liquid-air interface as we vary the magnet distance $L^{*}$. In the jetting experiments, we measure the critical magnet distance $L_{c}^{*}$ at which the magnetic hump transforms to a jet (see Fig. 2).

A DSLR camera (Nikon, Tokyo, Japan) is connected to a macro lens (Sigma Corp., Fukushima, Japan) via a macro bellows (Fotodiox, Waukegan, IL, USA) to take images of the static magnetic hump. A Phantom V9 fastcamera (Vision Research, Wayne, NJ, USA) is used in place of the DSLR when the hump-jet transition experiments are conducted to capture the jet formation. IMAGEJ software is used to process and analyse all experimental images. 


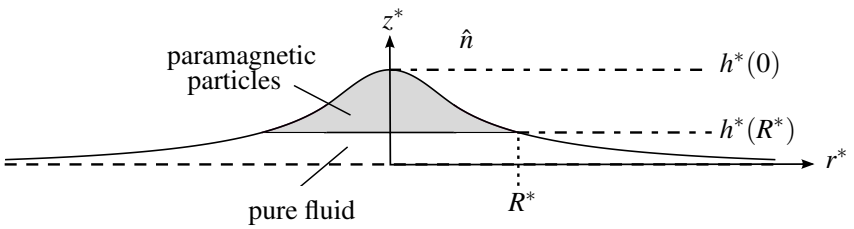

Fig. 3 Schematic illustrating the volume occupied by paramagnetic particles.

\section{Mathematical formulation}

\subsection{Governing equations for the magnetic deformation}

The system we study is distinguished from purely ferrofluid systems by the presence of a non-magnetic region of the fluid that surrounds the magnetic region. The magnetic region is defined as the volume where all of the magnetic particles are collected, while the non-magnetic region contains no magnetic particles. We choose to describe the system using cylindrical polar coordinates, $\left(r^{*}, \theta^{*}, z^{*}\right)$, and assume that the set-up is both stable and axisymmetric and thus independent of time and of angle, $\theta^{*}$. We assume that the paramagnetic particles are contained within a domain bounded by the upper surface, given by $z^{*}=h^{*}\left(r^{*}\right)$, and a horizontal base $z^{*}=h^{*}\left(R^{*}\right)$, so that $r^{*}=R^{*}$ represents the radial extent occupied by the paramagnetic particles, as illustrated in Fig. 3.

We begin our analysis with the non-magnetic region. Within this region the fluid is assumed to be non-magnetizable and so the fluid pressure, $p^{*}$, is simply hydrostatic,

$$
p^{*}=-\rho g z^{*} \text {. }
$$

Here $\rho$ is the fluid density and $g$ is the acceleration due to gravity, and we have set $p^{*}=0$ at $z^{*}=0$ (the reference of the flat interface) without loss of generality. We can account for the pressure drop across the interface and hydrostatic stresses by the Young-Laplace equation,

$$
p^{*}=2 \kappa^{*} \gamma,
$$

on $z^{*}=h^{*}$, where $\gamma$ is the interfacial tension in the nonmagnetic region and

$$
2 \kappa^{*}=\frac{\frac{\mathrm{d}^{2} h^{*}}{\mathrm{~d} r^{* 2}}+\frac{1}{r^{*}} \frac{\mathrm{d} h^{*}}{\mathrm{~d} r^{*}}+\frac{1}{r^{*}}\left(\frac{\mathrm{d} h^{*}}{\mathrm{~d} r^{*}}\right)^{3}}{\left(1+\left(\frac{\mathrm{d} h^{*}}{\mathrm{~d} r^{*}}\right)^{2}\right)^{3 / 2}}
$$

is the interfacial curvature. Replacing the pressure with its hydrostatic form (1) thus yields

$$
2 \kappa^{*} \gamma=-\rho g h^{*}\left(r^{*}\right)
$$

In the magnetic region, we treat the liquid as a ferrofluid that has a homogeneous magnetic susceptibility, $\chi$. We model the magnetic region using the standard ferrofluid equations applied to a static fluid of constant density equal to that in the non-magnetic region, which gives, ${ }^{12}$

$$
-\nabla^{*} p^{*}+\mu_{o} \chi \nabla^{*} H^{* 2}+\rho \boldsymbol{g}=\mathbf{0} .
$$

Here $\nabla^{*}$ denotes the dimensional gradient operator, $\mu_{o}=1.257 \times 10^{-6} \mathrm{~m} \mathrm{~kg} / \mathrm{s}^{2} \mathrm{~A}^{2}$ represents the permeability of free space, $\boldsymbol{g}=-g \hat{\boldsymbol{z}}$, where $\hat{\boldsymbol{z}}$ denotes the unit vector in the $z^{*}$ direction, and $H^{* 2}=\boldsymbol{H}^{*} \cdot \boldsymbol{H}^{*}$, where $\boldsymbol{H}^{*}$ is the magnetic field. In obtaining this result we have assumed that the fluid is linearly magnetizable so that the magnetization, $\boldsymbol{M}^{*}$, may be written as $\boldsymbol{M}^{*}=\chi \boldsymbol{H}^{*}$. Integration of the $z^{*}$-component of Eqn. (5) gives

$$
-p^{*}+\mu_{o} \chi H^{* 2}-\rho g^{*} z^{*}=c,
$$

where $c$ is a constant of integration. Within this magnetic region, the modified form of the Young-Laplace equation, ${ }^{12}$

$$
p^{*}+\frac{1}{2} \mu_{o} \chi^{2}\left(\hat{\boldsymbol{n}} \cdot \boldsymbol{H}^{*}\right)^{2}=2 \kappa^{*} \gamma,
$$

provides a condition at the interface, $z^{*}=h^{*}$, where $\hat{\boldsymbol{n}}$ is the unit outward normal vector to the interface (see Fig. 3). Note here we have made the experimentally justified assumption that the interfacial tension in the magnetic region is equal to that in the non-magnetic surrounding region. Using (6) and (7) allows us to eliminate $p^{*}$ to obtain

$$
2 \kappa^{*} \gamma+\rho g^{*} h^{*}=\mu_{o} \chi H^{* 2}+\frac{1}{2} \mu_{o} \chi^{2}\left(\hat{\boldsymbol{n}} \cdot H^{*}\right)^{2}-c .
$$

The constant $c$ is determined by matching the magnetic and non-magnetic regions at $r^{*}=R^{*}$, so that (8) becomes

$$
\begin{aligned}
2 \kappa^{*} \gamma+ & \rho g h^{*}=\mu_{o} \chi\left\{H^{* 2}-\left.H^{* 2}\right|_{r^{*}=R^{*}}\right\} \\
& +\frac{1}{2} \mu_{o} \chi^{2}\left\{\left[\hat{\boldsymbol{n}} \cdot \boldsymbol{H}^{*}\right]^{2}-\left.\left[\hat{\boldsymbol{n}} \cdot \boldsymbol{H}^{*}\right]^{2}\right|_{r^{*}=R^{*}}\right\} .
\end{aligned}
$$

Here the field, $\boldsymbol{H}^{*}$, for a spherical magnet, is given by a known solution of the magnetic field of a uniformly magnetized sphere, ${ }^{25}$ with a coordinate transformation to account for our $\left(r^{*}, z^{*}\right)$ cylindrical polar coordinate system,

$$
\begin{aligned}
\boldsymbol{H}^{*} & =\left\{\frac{M^{*} R_{m}^{* 3}\left(z^{*}-L^{*}\right) r^{*}}{\left[\left(L^{*}-z^{*}\right)^{2}+r^{* 2}\right]^{\frac{5}{2}}}\right\} \hat{\boldsymbol{r}} \\
& +\frac{1}{3}\left\{\frac{2 M^{*} R_{m}^{* 3}\left(z^{*}-L^{*}\right)^{2}}{\left[\left(L^{*}-z^{*}\right)^{2}+r^{* 2}\right]^{\frac{5}{2}}}-\frac{M^{*} R_{m}^{* 3} r^{* 2}}{\left[\left(L^{*}-z^{*}\right)^{2}+r^{* 2}\right]^{\frac{5}{2}}}\right\} \hat{\boldsymbol{z}}
\end{aligned}
$$


where $M^{*}$ is the magnet magnetization, $R_{m}^{*}$ is the radius of the magnet, and $\hat{r}$ denotes the unit vector in the $r^{*}$ direction.

The system (4) and (9) comprises two second-order equations and is solved by imposing four boundary conditions, namely zero gradient of the deformed interface at the centre of the hump due to symmetry, zero deflection of the fluid interface in the far field, and matching interface deflection and gradient at the point $r^{*}=R^{*}$ :

$$
\begin{array}{cc}
\frac{\mathrm{d} h^{*}(0)}{\mathrm{d} r^{*}}=0, & h^{*}\left(r^{*} \rightarrow \infty\right) \rightarrow 0, \\
h^{*}\left(R^{*-}\right)=h^{*}\left(R^{*+}\right), & \frac{\mathrm{d} h^{*}\left(R^{*-}\right)}{\mathrm{d} r^{*}}=\frac{\mathrm{d} h^{*}\left(R^{*+}\right)}{\mathrm{d} r^{*}} .
\end{array}
$$

For a given experiment, the paramagnetic beads occupy a volume, $V^{*}$, given by

$$
\int_{0}^{2 \pi} \int_{0}^{R^{*}} r^{*}\left(h^{*}\left(r^{*}\right)-h^{*}\left(R^{*}\right)\right) \mathrm{d} r^{*} \mathrm{~d} \theta^{*}=V^{*}
$$

as illustrated in Fig. 3. This equation determines the radial extent occupied by the paramagnetic particles, $R^{*}$, and closes the system.

\subsection{Non-dimensionalization}

We non-dimensionalize the system via

$$
h^{*}=h_{c}^{*} h, \quad r^{*}=R_{c}^{*} r, \quad R^{*}=R_{c}^{*} R,
$$

where

$$
h_{c}^{* 2}=\frac{\mu_{o} \chi M^{* 2} R_{m}^{* 6} V^{*}}{18 \pi \gamma L^{* 6}}, \quad R_{c}^{* 2}=\frac{V^{*}}{2 \pi h_{c}^{*}} .
$$

Here, $h_{c}^{*}$ is a characteristic interfacial deflection, and captures the dominant balance between the paramagnetic attraction and the surface tension, and $R_{c}^{*}$ expresses the typical radial spread of the paramagnetic material.

Substitution of the expression for the magnetic field, Eqn. (10), into Eqn. (9) and employing the non- dimensionalization (13) leads to

$$
\begin{gathered}
\frac{h^{\prime \prime}+\frac{1}{r} h^{\prime}+\frac{\varepsilon^{2}}{\alpha^{2}} \frac{1}{r} h^{\prime 3}}{\left[1+\frac{\varepsilon^{2}}{\alpha^{2}} h^{\prime 2}\right]^{\frac{3}{2}}-B h=\left[\frac{4(\varepsilon h-1)^{2}+\alpha^{2} r^{2}}{\left((\varepsilon h-1)^{2}+\alpha^{2} r^{2}\right)^{4}}\right.} \\
\left.-\frac{4(\varepsilon h(R)-1)^{2}+\alpha^{2} R^{2}}{\left((\varepsilon h(R)-1)^{2}+\alpha^{2} R^{2}\right)^{4}}\right] \\
+\frac{\chi}{2}\left[\frac{9(1-\varepsilon h)^{2} \alpha^{4} r^{2}+\varepsilon^{2}\left(h^{\prime}\right)^{2}\left(2(1-\varepsilon h)+\alpha^{2} r^{2}\right)^{2}}{\left((1-\varepsilon h)^{2}+\alpha^{2} r^{2}\right)^{5}\left(\alpha^{2}+\varepsilon^{2}\left(h^{\prime}\right)^{2}\right)}\right. \\
\left.-\frac{9(1-\varepsilon h(R))^{2} \alpha^{4} R^{2}+\varepsilon^{2}\left(h^{\prime}(R)\right)^{2}\left(2(1-\varepsilon h(R))+\alpha^{2} R^{2}\right)^{2}}{\left((1-\varepsilon h(R))^{2}+\alpha^{2} R^{2}\right)^{5}\left(\alpha^{2}+\varepsilon^{2}\left(h^{\prime}(R)\right)^{2}\right)}\right],
\end{gathered}
$$

for $r \leq R$, and,

$$
\frac{h^{\prime \prime}+\frac{1}{r} h^{\prime}+\frac{\varepsilon^{2}}{\alpha^{2}} \frac{1}{r} h^{\prime 3}}{\left[1+\frac{\varepsilon^{2}}{\alpha^{2}} h^{\prime 2}\right]^{\frac{3}{2}}}-B h=0,
$$

for $r \geq R$, where primes denote differentiation. The system is thus characterized by three dimensionless parameters: the Bond number

$$
B=\frac{\rho g R_{c}^{* 2}}{\gamma}=\frac{3 \rho g L^{* 3}}{M^{*} R_{m}^{* 3}} \sqrt{\frac{V^{*}}{2 \pi \mu_{o} \chi \gamma}},
$$

which represents the ratio of buoyancy to surface tension;

$$
\alpha=\frac{R_{c}^{*}}{L^{*}}=\left(\frac{9 \gamma L^{* 2} V^{*}}{2 \pi \mu_{o} \chi M^{* 2} R_{m}^{* 6}}\right)^{1 / 4}
$$

which denotes the ratio of the typical radial spread to magnet distance, and provides a representation of the total volume of magnetic material; and

$$
\varepsilon=\frac{h_{c}^{*}}{L^{*}}=\sqrt{\frac{\mu_{o} \chi M^{* 2} R_{m}^{* 6} V^{*}}{18 \pi \gamma L^{* 8}}}
$$

which denotes the aspect ratio of the hump. For all of the experiments conducted, we find that, for stable interfacial profiles, $\varepsilon$ is always small, with the fluid jetting well before an order-one aspect ratio of the interfacial profile can be achieved. We exploit this feature in an asymptotic analysis in $\S 4$.

4 | Journal Name, 2010, [vol],1-10

This journal is @ The Royal Society of Chemistry [year] 

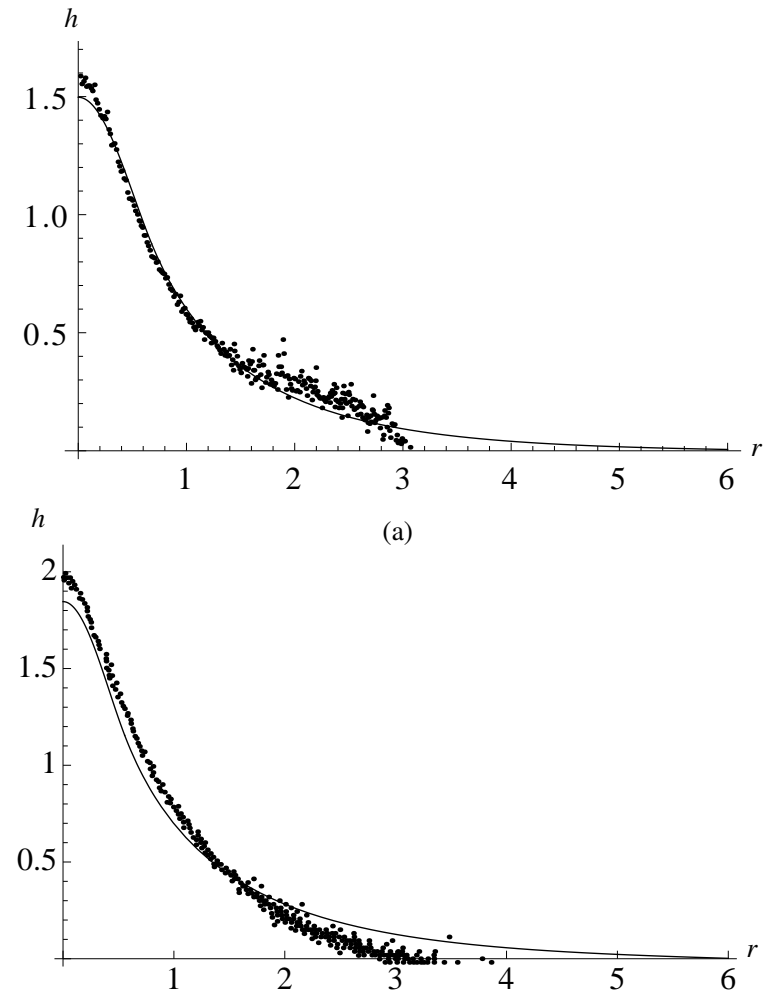

(b)

Fig. 4 Comparison of the numerical solution to Eqns. (15) subject to (19) and (20) (solid lines) with experimental data (points). After depositing the $1 \mathrm{~mL}$ of water and $1 \mu \mathrm{L}$ of ferrofluid on the substrate, the magnet is brought to the vicinity of the mixture until a static shape is achieved. Results from two static magnetic deformation profiles with $R_{m}^{*}=3.175 \mathrm{~mm}, \gamma=0.07 \mathrm{Nm}^{-1}$, and $V^{*} \approx 0.47 \mathrm{~mm}^{3}$ are shown: (a) $L^{*} \approx 6.33 \mathrm{~mm}$, giving $\alpha \approx 0.28, B \approx 0.45$ and $\varepsilon \approx 0.015$; (b) $L^{*} \approx 5.65 \mathrm{~mm}$, giving $\alpha \approx 0.27, B \approx 0.32$, and $\varepsilon \approx 0.024$. The results show excellent agreement between the experiments and the numerical prediction.

The dimensionless counterparts to the boundary conditions (11) read

$$
\begin{gathered}
h^{\prime}(0)=0, \quad h(r \rightarrow \infty)=0, \\
h\left(R^{-}\right)=h\left(R^{+}\right), \quad h^{\prime}\left(R^{-}\right)=h^{\prime}\left(R^{+}\right) .
\end{gathered}
$$

The dimensionless radial spread of the paramagnetic material, $R$, which appears in $(15 a)$ and $(19 c, d)$ is also unknown a priori, and is determined by satisfying the dimensionless version of (12) that enforces the volume conservation of paramagnetic beads,

$$
\int_{0}^{R} r h(r) \mathrm{d} r=1+\frac{1}{2} R^{2} h(R) .
$$

The numerical solution to Eqns. (15) is obtained using an Adams-Bashforth multistep method, and solving for the ap- propriate value of $h(0)$ that satisfies the volume constraint (20). The results are compared in Fig. 4 with data obtained for an experiment where $1 \mathrm{~mL}$ of water and $1 \mu \mathrm{L}$ of ferrofluid are placed on the substrate and in the region of influence of the magnetic field until a static hump shape is achieved. As the magnet is brought closer to the free surface, the deflection of the height of the magnetic hump increases.

In our comparison with the experimental data, we fit only once, for the magnetic product $\chi M^{* 2}$, after which the numerical system is then fully determined. The calculation determines the magnitude of the characteristic hump height, $h_{c}$. In this experiment, we find that $\chi M^{* 2} \approx 9.6 \times 10^{8} \mathrm{~A}^{2} / \mathrm{m}^{2}$. Typically $\chi \approx O\left(10^{-3}\right)$ and $M^{*} \approx O\left(10^{6} \mathrm{~A} / \mathrm{m}\right)$. The results show extremely good agreement between the numerical solutions and the experiments. Specifically, we observe that the numerical solution is able to capture not only the height and width of the hump, but the inflection in the interfacial profile.

Whilst the numerical solution is in excellent agreement with the experimental data, the iterative procedure employed to determine the solution is somewhat arduous and, moreover, it is difficult to use this to gain insight into the parametric dependence of the system behaviour. With this observation in mind we proceed in the following section to seek a simplified analytic solution for the interfacial profile, by exploiting the typically small ratio of vertical deflection of the fluid surface to the horizontal extent occupied by the paramagnetic particles, that is, $\varepsilon$, and the smallness of the magnetic susceptibility, $\chi$.

\section{An asymptotic approximation to the interfa- cial profile}

The interfacial curvature is generally small when the system is in an equilibrium configuration prior to the jetting of the fluid, which manifests itself in the smallness of the parameter $\varepsilon$. We may exploit this feature by analysing the governing equations (15) and boundary conditions (19) and (20) asymptotically in the limit $\varepsilon \rightarrow 0$. In addition, the magnetic susceptibility, $\chi$, is also typically small, of the order $10^{-3}$. Thus, we neglect terms premultiplied by $\varepsilon$ or $\chi$ in (15) to obtain the simplified linear system for $h$,

$$
\begin{array}{lll}
h^{\prime \prime}+\frac{1}{r} h^{\prime}-B h=\frac{4+\alpha^{2}}{\left(1+\alpha^{2}\right)^{4}}-\frac{4+\alpha^{2} r^{2}}{\left(1+\alpha^{2} r^{2}\right)^{4}}, & r \leq R, \\
h^{\prime \prime}+\frac{1}{r} h^{\prime}-B h=0, & r \geq R,
\end{array}
$$

subject to the five boundary conditions

$$
\begin{gathered}
h^{\prime}(0)=0, \quad h\left(R^{-}\right)=h\left(R^{+}\right), \quad h^{\prime}\left(R^{-}\right)=h^{\prime}\left(R^{+}\right), \\
h(r \rightarrow \infty)=0, \quad \int_{0}^{1} r h(r) \mathrm{d} r=1+\frac{1}{2} R^{2} h(R) .
\end{gathered}
$$


This boundary value problem now depends only on two dimensionless parameters, namely $\alpha$ and $B$.

We first solve $(21 b)$ subject to $(22 d)$ to yield

$$
h(r)=A K_{0}(\sqrt{B} r)
$$

where $K_{0}$ denotes the modified Bessel function of the second kind and $A$ is a constant to be determined. Manipulation of (23) and use of $(22 b, c)$ provides a Neumann boundary condition satisfied by the profile for $r \leq R$,

$$
\sqrt{B} K_{1}(\sqrt{B} R) h(R)+K_{0}(\sqrt{B} R) h^{\prime}(R)=0 .
$$

We are then left to solve the decoupled problem (21a) subject to (22a) and (24). This system may be solved via the method of variation of parameters to provide the height profile, $h$, for $r \leq R,{ }^{26}$

$$
\begin{aligned}
h & =K_{0}(\sqrt{B} r) \int_{0}^{r} s I_{0}(\sqrt{B} s)\left(\frac{4+\alpha^{2} s^{2}}{\left(1+\alpha^{2} s^{2}\right)^{4}}-\frac{4+\alpha^{2} R^{2}}{\left(1+\alpha^{2} R^{2}\right)^{4}}\right) \mathrm{d} s \\
& +I_{0}(\sqrt{B} r) \int_{r}^{R} s K_{0}(\sqrt{B} s)\left(\frac{4+\alpha^{2} s^{2}}{\left(1+\alpha^{2} s^{2}\right)^{4}}-\frac{4+\alpha^{2} R^{2}}{\left(1+\alpha^{2} R^{2}\right)^{4}}\right) \mathrm{d} s .
\end{aligned}
$$

The constant $A$ may then be determined using (22b) to give the profile for $r \geq R$,

$$
\begin{aligned}
h(r)= & K_{0}(\sqrt{B} r) \\
& \times \int_{0}^{R} s I_{0}(\sqrt{B} s)\left(\frac{4+\alpha^{2} s^{2}}{\left(1+\alpha^{2} s^{2}\right)^{4}}-\frac{4+\alpha^{2} R^{2}}{\left(1+\alpha^{2} R^{2}\right)^{4}}\right) \mathrm{d} s .
\end{aligned}
$$

Finally, the parameter $R$ is determined by conservation of volume, $(22 e)$, giving the integral relation

$$
\begin{gathered}
\left(\sqrt{B} K_{0}(\sqrt{B} R)+R K_{1}(\sqrt{B} R)\right) \\
\times \int_{0}^{R} s I_{0}(\sqrt{B} s)\left(\frac{4+\alpha^{2} s^{2}}{\left(1+\alpha^{2} s^{2}\right)^{4}}-\frac{4+\alpha^{2} R^{2}}{\left(1+\alpha^{2} R^{2}\right)^{4}}\right) \mathrm{d} s \\
=\frac{3 R^{4} \alpha^{2}\left(5+4 R^{2} \alpha^{2}+R^{4} \alpha^{4}\right)}{4 \sqrt{B}\left(1+R^{2} \alpha^{2}\right)^{4}}-\sqrt{B}
\end{gathered}
$$

The analytic solution for the magnetic hump profile, $(25 a)$ is shown in Fig. 5, for the same experimental set-up as presented in Fig. 4(a). We observe an outstanding agreement between the asymptotic solution and the full numerical solution to (15) subject to (19) and (20). This success motivates us to utilize the asymptotic model (25) and (26) to study in more detail the dependence of the interfacial profile on the key physical parameters.

Of particular interest is the behaviour of a given experimental set-up upon variations in the position of the magnet from

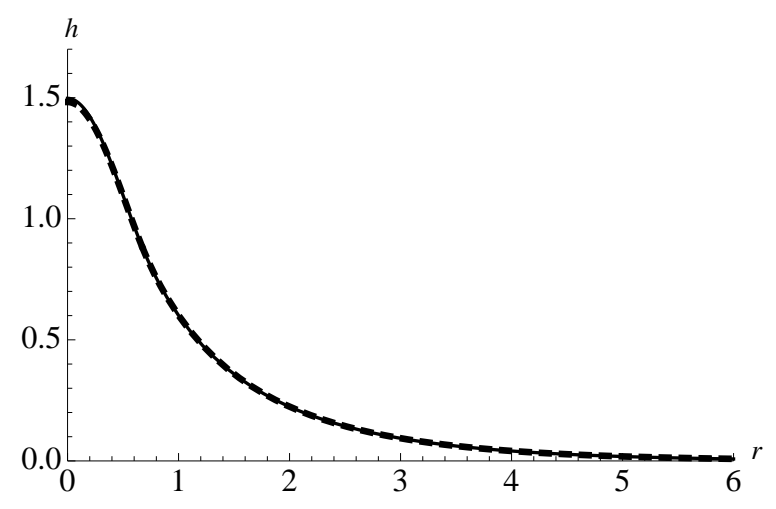

Fig. 5 Comparison of the numerical solution to Eqn. (15) subject to (19) and (20) (solid line) and the analytic asymptotic result (25a) and (25b) (dashed line) for the same experimental set-up as shown in Fig. 4(a), illustrating an outstanding agreement.

the fluid in its undeformed state, $L^{*}$. To understand this behaviour it is helpful to restate the mathematical model in terms of the following new parameters:

$$
\mathscr{B}=\frac{\rho g R_{m}^{* 2}}{\gamma}, \quad \mathscr{A}=\frac{2 \pi \chi \mu_{o} M^{2}}{9 \rho g R_{m}^{*}}, \quad \mathscr{L}=\frac{L^{*}}{R_{m}^{*}}, \quad \mathscr{V}=\frac{V^{*}}{R_{m}^{3}},
$$

Assuming that the size of the external magnet is fixed, variations in: $\mathscr{B}$ capture changes in the surface tension of the fluid; $\mathscr{A}$ provides a dimensionless representation of the strength of the external magnet; $\mathscr{L}$ gives the dimensionless distance between the magnet centre and the fluid interface in its undisturbed state; and $\mathscr{V}$ provides a dimensionless measure of the volume of paramagnetic particles contained within the fluid. In terms of these parameters,

$$
B=\left(\frac{\mathscr{B} \mathscr{V} \mathscr{L}^{6}}{\mathscr{A}}\right)^{1 / 2}, \quad \alpha=\left(\frac{\mathscr{L}^{2} \mathscr{V}}{\mathscr{A}}\right)^{1 / 4}
$$

It is also appropriate to rescale $R$ and $h$ via

$$
\begin{aligned}
& R=\frac{R_{m}^{*}}{R_{c}^{*}} \mathscr{R}=\left(\frac{\mathscr{L}^{6}}{4 \mathscr{A} \mathscr{V} \mathscr{B}}\right)^{1 / 6} \mathscr{R}, \\
& h=\frac{R_{m}^{*}}{h_{c}^{*}} \mathscr{H}=\left(\frac{\mathscr{A} \mathscr{B} \mathscr{B}}{\pi^{2} \mathscr{L}}\right)^{1 / 2} \mathscr{H} .
\end{aligned}
$$

Despite no longer being in its most compact form, expressing the system in terms of these new variables is helpful in characterizing the behaviour with respect to the variables that we are most easily able to adjust experimentally.

As the distance between the external magnet and the fluid is increased, by increasing $\mathscr{L}$, the radial extent over which the paramagnetic particles occupy, $\mathscr{R}$, may be determined using Eq. (26), with the appropriate parameter substitutions (28). 
As we would expect, this radial extent increases with increasing distance between the magnet and the fluid, as the hump becomes less pronounced and the particles spread out (Fig. 6). However, interestingly, we identify a critical finite distance, $\mathscr{L}_{c}$, at which point $\mathscr{R} \rightarrow \infty$. For $\mathscr{L}>\mathscr{L}_{c}$ no such solution for $\mathscr{R}$ exists. We notice that $\mathscr{L}_{c}$ depends on $\mathscr{A}$ and $\mathscr{L}$ but not on $\mathscr{B}$ (shown in Fig. 6). We may identify the dependence of $\mathscr{L}_{c}$ on the system parameters by taking the limit as $R \rightarrow \infty$ in (26). In this limit, the integral component tends to zero and we are left with the simple relationship

$$
\mathscr{L}_{c}=\left(\frac{3}{4}\right)^{1 / 8}\left(\frac{\mathscr{A}}{\mathscr{V}}\right)^{1 / 4} .
$$

When the distance between the magnet and the fluid exceeds this critical value, our assumption that the paramagnetic particles are contained within the hump generated by the magnetic field (that is, above $z=0$ ) no longer holds, and instead the particles now become dispersed into the bulk solution, beneath $z=0$. Since the ability to collect particles is not influenced by the shape of the interface this offers an explanation for the independence of the critical separation, $\mathscr{L}_{c}$, on surface tension (i.e., $\mathscr{B}$ ). The relationship (30) thus provides a quantitative prediction of when the particles are constrained to a localized region by the external magnetic field. Such a prediction may prove useful in scenarios in which containment of particulates is important, such as in water purification applications.

For any of the given configurations described above, we may determine the corresponding maximum interfacial deflection, at the centre of the hump. Eqn. (25a) gives a maximum dimensionless interfacial deflection of

$$
\begin{aligned}
h_{\max } & =h(0) \\
& =\int_{0}^{R} s K_{0}(\sqrt{B} s)\left(\frac{4+\alpha^{2} s^{2}}{\left(1+\alpha^{2} s^{2}\right)^{4}}-\frac{4+\alpha^{2} R^{2}}{\left(1+\alpha^{2} R^{2}\right)^{4}}\right) \mathrm{d} s,
\end{aligned}
$$

which may be used to determine $\mathscr{H}_{\max }=\mathscr{H}(0)$ using (28) and (29).

The result (31) provides a simple expression for the maximum interfacial deflection for a given experimental set-up and allows for ready graphical visualization (Fig. 7). Here we see that the interfacial deflection increases with increasing magnetic field strength (increasing $\mathscr{A}$ ), decreasing surface tension (increasing $\mathscr{B}$ ), and increasing volume of magnetic material (increasing $\mathscr{V}$ ). We compare in Fig. 8 the predictions of this simple formula with experimental data. Here, the external magnet is moved successively closer to the interface and the maximum deflection is recorded, until the surface tension of the fluid can no longer constrain the paramagnetic particles and jetting occurs. The general trend of the experimental data is shown to be captured well by the theory for the ten data points recorded at the furthest distances between

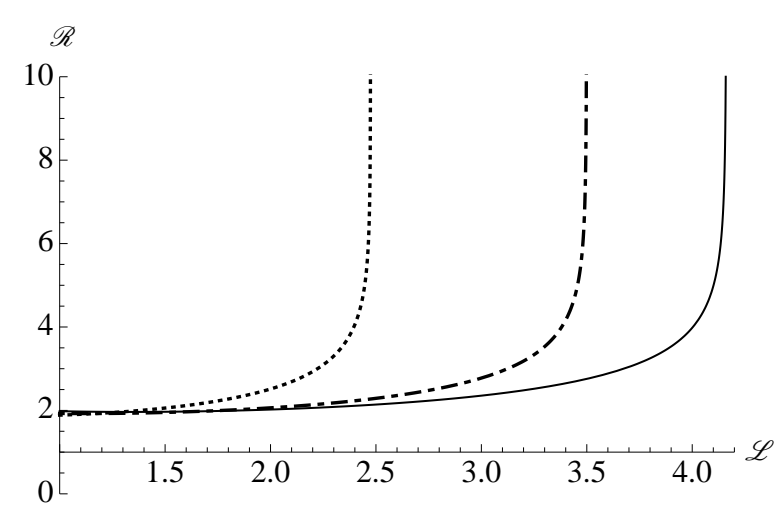

(a)

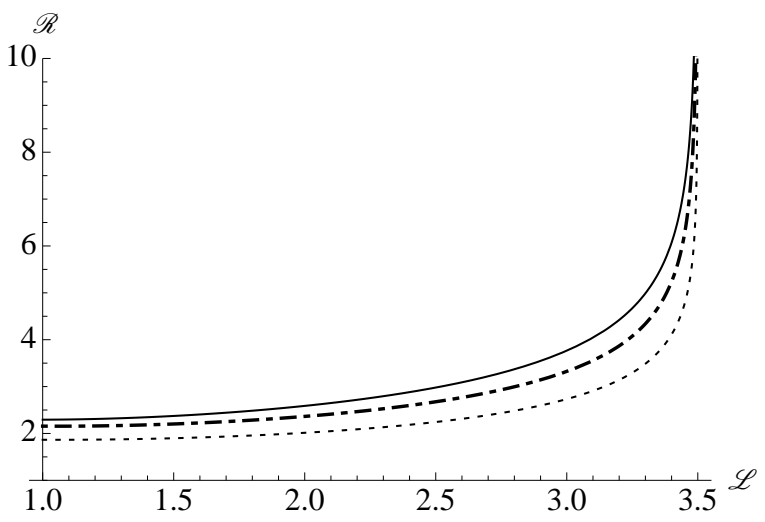

(b)

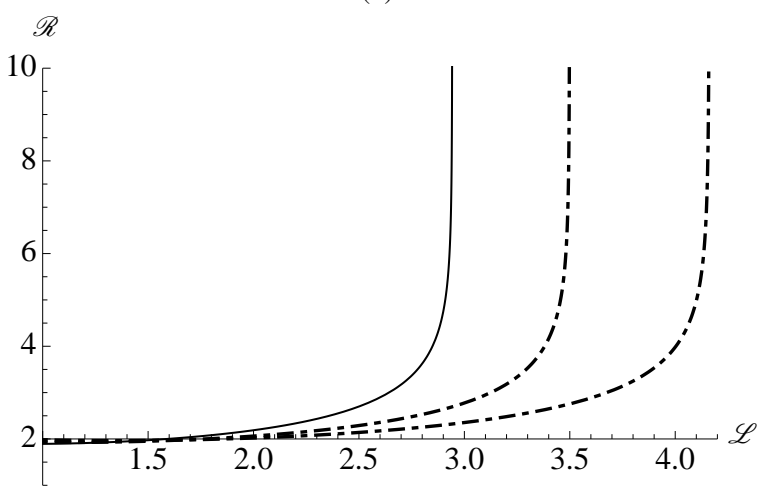

(c)

Fig. 6 Dependence of the radial extent, $\mathscr{R}$, occupied by the paramagnetic particles on distance between the external magnet and the fluid, $\mathscr{L}$ for (a) $\mathscr{A}=5$ (dashed), 20 (dot-dashed), 40 (solid), with $\mathscr{B}=1$ and $\mathscr{V}=0.1$; (b) $\mathscr{B}=0.5$ (dashed), 5 (dot-dashed), 10 (solid), with $\mathscr{A}=20$ and $\mathscr{V}=0.1$; (c) $\mathscr{V}=0.05$ (dashed), 0.1 (dot-dashed), 0.2 (solid), with $\mathscr{A}=20$ and $\mathscr{B}=1$. We observe that $\mathscr{R} \rightarrow \infty$ at a finite value of $\mathscr{L}$, given by (30). 
the magnet and the interface before we observe a deviation, with the model predicting a smaller deflection than that observed experimentally. The position at which this deviation occurs is also identified with an apparent transition in the system behaviour, whereby the magnitude of the deflection increases much more rapidly as the magnet is moved successively closer. We postulate that it is at this transition point that the interface becomes unstable, which leads to the difference between experimental observation and theoretical predication. Indeed, experimental observations show that, as the magnet approaches the fluid it becomes increasingly difficult to ascertain when a steady profile exists: the profile is often observed to jet several tens of minutes after moving the external magnet an increment closer. This observation further reinforces the hypothesis that profiles in the region where theoretical prediction and experimental observation deviate are indeed unstable.

\section{Scaling model for the jetting transition}

In this final section we utilize the theory developed so far to extract a scaling law to predict the parametric regimes under which jetting occurs. Recall from $\S 3.2$ we identified the natural scaling for the interfacial deflection,

$$
h_{c}^{* 2}=\frac{\mu_{o} \chi M^{* 2} R_{m}^{* 6} V^{*}}{18 \pi \gamma L^{* 6}},
$$

that captures the dominant balance between the paramagnetic attraction and the surface tension. Experimental observations suggest that the values of the interfacial deflection and the radial extent occupied by the paramagnetic particles do not vary significantly for different experimental configurations just prior to jetting. Motivated by these characteristics we assume that the parameter $h_{c}^{*}$ in (32) may be treated as approximately constant at the point of jetting for any given experiment. Eqn. (32) then determines an approximate scaling relationship between the remaining parameters that we vary in our experiments, namely $R_{m}^{*}, \gamma$, and the critical distance between the external magnet and the unperturbed surface below which jetting occurs, $L_{c}^{*}$ :

$$
L_{c}^{*} \approx C \frac{R_{m}^{*}}{\gamma^{\frac{1}{6}}}
$$

where $C$ is a dimensional scaling constant that is set by the variables we have fixed in our experiments.

Fig. 9(a) shows the critical magnet distance, $L_{c}^{*}$, for the jetting transition, plotted with varying external magnet radii and interfacial tensions in the water-air system (achieved via the addition of surfactant SDS, as described in §2). In general, increasing the interfacial tension results in a lower threshold distance $L_{c}^{*}$, as larger magnetic forces are required to destabilize the magnetic hump. Similarly, using a smaller magnet to produce a magnetic jet requires the magnet to be placed closer

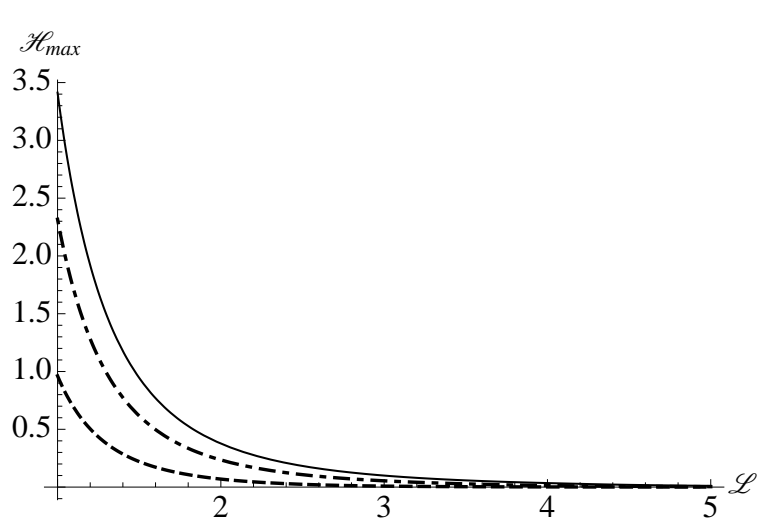

(a)

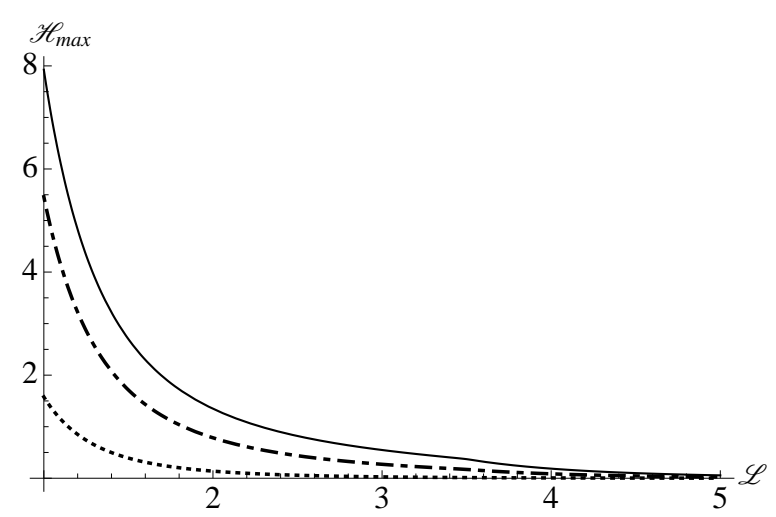

(b)

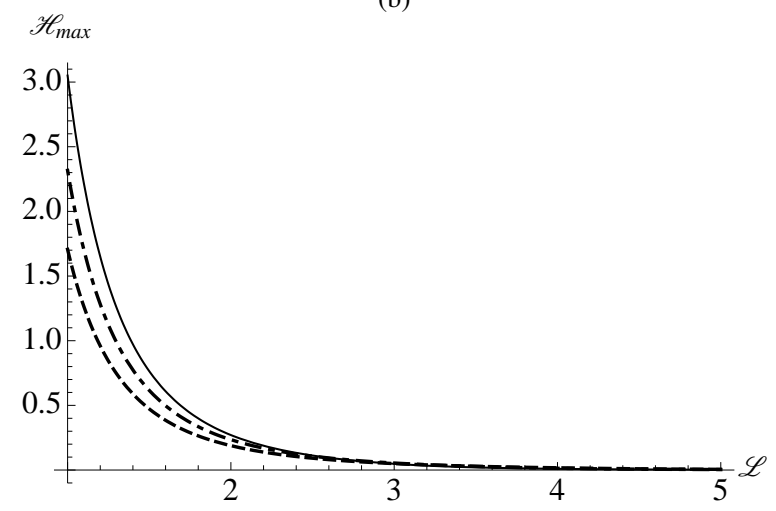

(c)

Fig. 7 The maximum interfacial deflection, $\mathscr{H}_{\max }=\mathscr{H}(0)$, determined using (29), (28) and (31), versus $\mathscr{L}$ for (a) $\mathscr{A}=5$ (dashed), 20 (dot-dashed) 40 (solid), with $\mathscr{B}=1$ and $\mathscr{V}=0.1$; (b) $\mathscr{B}=0.5$ (dashed), 5 (dot-dashed) 10 (solid), with $\mathscr{A}=20$ and $\mathscr{V}=0.1 ;$ (c) $\mathscr{V}=0.05$ (dashed), 0.1 (dot-dashed) 0.2 (solid), with $\mathscr{A}=20$ and $\mathscr{B}=1$. 


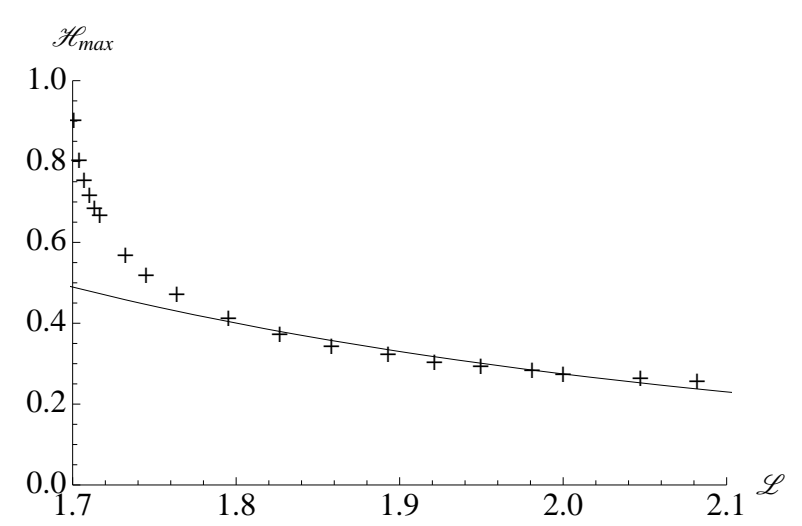

Fig. 8 The maximum interfacial deflection, $\mathscr{H}_{\max }=\mathscr{H}(0)$, determined using (29) and (31), versus $\mathscr{L}$ (solid line) compared with data for the experimental set-up with $R_{m}^{*}=3.17 \mathrm{~mm}, \gamma=0.07 \mathrm{~N} / \mathrm{m}$, and $V^{*}=0.47 \mathrm{~mm}^{3}$, giving $\mathscr{A} \approx 27, \mathscr{B} \approx 1.4$, and $\mathscr{V}=0.093$.
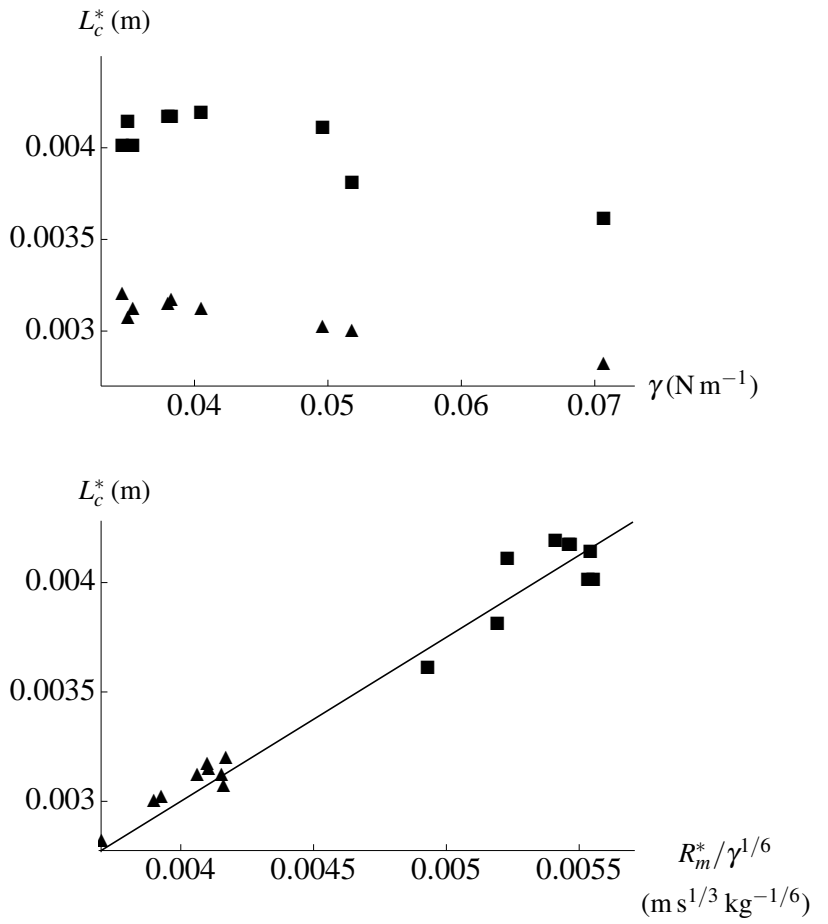

Fig. 9 (a) The experimental threshold magnet distance for the hump-jet transition, $L_{c}^{*}$, for two different magnet sizes $R_{m}^{*}=2.38 \mathrm{~mm}$ (triangles), $R_{m}^{*}=3.17 \mathrm{~mm}$ (squares), with $V^{*} \approx 0.47 \mathrm{~mm}^{3}$, plotted against the liquid-air interfacial tension, $\gamma$; (b) $L_{c}^{*}$ plotted against $R_{m}^{*} / \gamma^{1 / 6}$ for the data presented in (a), and the scaling fit $L_{c}^{*}=C R_{m}^{*} / \gamma^{1 / 6}$ with $\mathrm{C}=0.75$. to the interface before jetting is observed. In Fig. 9(b) the critical distance for jetting, $L_{c}^{*}$ is replotted versus the parametric group $R_{m}^{*} / \gamma^{* 1 / 6}$ predicted by our scaling law (33). The data is shown to collapse well onto this predicted scaling law. Thus, we may conclude that the scaling choice made for the numerical model in $\S 3$ provides the appropriate balance between the competing effects that constitute the development of an instability in the fluid interface and a transition to jetting, and gives a simple prediction for when jetting will occur.

\section{Conclusions}

In this paper we have studied the experimental results of the deformation of a free surface by an aggregate of magnetic particles. The system studied possesses a clear distinction from a conventional ferrofluid systems since it comprises both regions rich with magnetic material as well as regions of negligible magnetic content. The deflection of the interface arises as a result of the magnetic field induced by a spherical permanent magnet held above the fluid. This force causes the magnetic particles to collect at the liquid-air interface and deform the free surface. The result is a hump that is composed of both magnetic and non-magnetic regions due to the particle accumulation.

In addition, we derived a mathematical model that describes the behaviour of the magnetic-particle-laden fluid and the particle-free fluid regions. The model predicts the shape of the interface observed experimentally, capturing the main experimental measurements including the height of the hump and radial spread of the particles and the characteristic shape of the interface.

We used the smallness of the interfacial curvature and magnetic susceptibility to derive an asymptotic model whose results were almost indistinguishable from the predictions of the full numerical system. The reduced model enabled a detailed analysis of the dependence of the system behaviour on the key variables. In particular, this theory provided an implicit integral relation that determines the radial spread of the paramagnetic particles, as well as an explicit result for the maximum interfacial deflection. The former result led to a novel prediction for the critical distance of the external magnet from the fluid interface, beyond which, the paramagnetic particles are no longer enclosed within the hump, but instead are dispersed into the bulk fluid. The results of the asymptotic model were compared with experimental data for the deflection of the fluid interface as the external magnet is brought successively closer to the fluid. The predictions were shown to agree for distances between the magnet and the fluid that exceed a critical value. Below this value we postulated that the deviation in prediction and theory may be indicative of the development of an interfacial instability. 
We concluded by deriving a theoretical scaling law that predicts the transition to jetting that is observed when the external magnet is closer than a critical distance from the fluid. The prediction allowed us to collapse data from a series of independent experiments onto a single power law.

The results of this paper provide simple expressions that can be used to determine the typical deflection of a fluid containing paramagnetic particles, in the presence of an external magnet, the radial extent that these particles pervade, and the transition of the fluid to jetting and removal of the paramagnetic material. Such results may be useful in more complicated configurations where the control of particle transport and removal using magnetic separation is important, such as in the removal of heavy metals and the purification of water, or in drug delivery.

\section{Acknowledements}

This publication is based on work partially supported by Award No. KUK-C1-013-04, made by King Abdullah University of Science and Technology (KAUST). SSHT acknowledges the National Science Foundation (NSF-CBET-0961081) for support. The authors acknowledge Janine and Joel Nunes for the construction of the motorized positioner used in these experiments.

\section{References}

1 R. S. Molday, S. P. Yen and A. Rembaum, Nature, 1977, 268, 437-438.

2 S. Miltenyi, W. Mller, W. Weichel and A. Radbruch, Cytometry, 1990, 11, 231-238.

3 M. Puig-De-Morales, M. Grabulosa, J. Alcaraz, J. Mullol, G. N. Maksym, J. J. Fredberg and D. Navajas, J. Appl. Physiol., 2001, 91, 1152-1159.

4 A. R. Bausch, U. Hellerer, M. Essler, M. Aepfelbacher and E. Sackmann, Biophys. J., 2001, 80, 2649-2657.

5 C. Wilhelm, F. Gazeau and J.-C. Bacri, Phys. Rev. E, 2003, 67, 061908.

6 N. Xia, T. P. Hunt, B. T. Mayers, E. Alsberg, G. M. Whitesides, R. M. Westervelt and D. E. Ingber, Biomed. Microdevices, 2006, 8, 299-308.

7 N. Pamme and C. Wilhelm, Lab Chip, 2006, 6, 974-980.

8 J. D. Adams, U. Kim and H. T. Soh, Proc. Natl. Acad. Sci., 2008, 105, 18165-18170.

9 C. W. Yung, J. Fiering, A. J. Mueller and D. E. Ingber, Lab Chip, 2009, 9, 1171-1177.

10 S. S. H. Tsai, J. S. Wexler, J. Wan and H. A. Stone, Lab Chip, 2013, 13, 119-125.

11 S. S. H. Tsai, J. S. Wexler, J. Wan and H. A. Stone, Appl. Phys. Lett., 2011, 99, 153509-3.

12 R. E. Rosensweig, Annu. Rev. Fluid Mech., 1987, 19, 437-461.

13 A. Engel, A. Lange, H. Langer, T. Mahr and M. V. Chetverikov, J. Magn. Magn. Mater., 1999, 201, 310-312.

14 A. Lange, H. Langer and A. Engel, Physica D, 2000, 140, 294-305.

15 R. Friedrichs and A. Engel, Eur. Phys. J. B, 2000, 18, 329-335.

16 B. Abou, J. Wesfreid and S. Roux, J. Fluid Mech., 2000, 416, 217-237.

17 H. E. Potts and D. A. Diver, New J. Phys., 2001, 3, 7.17.14.

18 C. Gollwitzer, G. Matthies, R. Richter, I. Rehberg and L. Tobiska, J. Fluid Mech., 2007, 571, 455-474.
19 K. Raj, B. Moskowitz and R. Casciari, J. Magn. Magn. Mater., 1995, 149, 174-180.

20 T. Jamin, C. Py and E. Falcon, Phys. Rev. Lett., 2011, 107, 204503.

21 F. N. Pirmoradi, J. K. Jackson, H. M. Burt and M. Chiao, Lab Chip, 2011, 11, 3072-3080.

22 M. A. Shannon, P. W. Bohn, M. Elimelech, J. G. Georgiadis, B. J. Marias and A. M. Mayes, Nature, 2008, 452, 301-310.

23 S. Yean, L. Cong, C. T. Yavuz, J. T. Mayo, W. W. Yu, A. T. Kan, V. L. Colvin and M. B. Tomson, J. Mater. Res., 2005, 20, 3255-3264.

24 C. T. Yavuz, J. T. Mayo, W. W. Yu, A. Prakash, J. C. Falkner, S. Yean, L. Cong, H. J. Shipley, A. Kan, M. Tomson, D. Natelson and V. L. Colvin, Science, 2006, 314, 964-967.

25 D. J. Griffiths, Introduction to Electrodynamics, Prentice Hall New Jersey, 2012, vol. 4.

26 D. Quinn, J. Feng and H. A. Stone, Langmuir, 2013, 29, 14271434.

10 | Journal Name, 2010, [vol], 1-10

This journal is @ The Royal Society of Chemistry [year] 



\section{RECENT REPORTS}

13/10 Capillary deformations of bendable films

Schroll

Adda-Bedia

Cerda

Huang

Menon

Russell

Toga

Vella

Davidovitch

13/11 Twist and stretch of helices: All you need is Love

Đuričković

Goriely

Maddocks

13/12 Switch on, switch off: stiction in nanoelectromechanical switches

Wagner

Vella

13/13 Pinning, de-pinning and re-pinning of a slowly varying rivulet

Paterson

Wilson

Duffy

13/14 Travelling-wave similarity solutions for a steadily translating slender dry patch in a thin fluid film

Yatim

Duffy

Wilson

13/15 A stochastic model for early placental development

Cotter

Klika

Kimpton

Collins

Heazell

13/16 Experimentally-calibrated population of models predicts and ex-

Britton plains inter-subject variability in cardiac cellular electrophysiology

Bueno-Orovio

Van Ammel

Lu

Towart

Gallacher

Rodriguez

13/17 Elastometry of deflated capsules elastic moduli from shape and wrinkle analysis

Knoche

Vella

Aumaitre

Degen

Rehage

Cicuta

Kierfeld

13/18 The effect of a concentration-dependent viscosity on particle

Herterich transport in a channel flow with porous walls

Griffiths

Field

Vella

13/19 On a poroviscoelastic model for cell crawling

Kimpton

Whiteley

Waters

Oliver 
13/26 The counterbend phenomenon: a generic property of the axoneme and cross-linked filament bundles

Gadêlha

Gaffney

Goriely

13/27 A well-posedness framework for inpainting based on coherence

März transport

13/28 Minimizing synchronizations in sparse iterative solvers for dis-

Zhu

tributed supercomputers

$\mathrm{Gu}$

Liu

13/29 Computational probabilistic quantification of pro-arrhythmic risk

Wallman from scar and left-to-right heterogeneity in the human ventricles

Bueno-Orovio

Rodriguez

13/30 Population of human ventricular cell models calibrated with in vivo

Zhou measurements unravels ionic mechanisms of cardiac alternans

Bueno-Orovio

Orini

Hanson

Haywood

Taggart

Lambiase

Burrage

Rodriguez

13/31 On the eigenvalues and eigenvectors of nonsymmetric saddle

Pestana point matrices preconditioned by block triangular matrices

13/32 Singular inextensible limit in the vibrations of post-buckled rods: Neukirch analytical derivation and role of boundary conditions

Goriely

Thomas

Copies of these, and any other OCCAM reports can be obtained from:

\author{
Oxford Centre for Collaborative Applied Mathematics \\ Mathematical Institute \\ 24 - 29 St Giles' \\ Oxford \\ OX1 3LB \\ England \\ www.maths.ox.ac.uk/occam
}

